\title{
L'évolution de la répression judiciaire des abus sexuels commis par des clercs sur les personnes en situation de vulnérabilité (1950-2020)
}

\section{Thomas Boullu}

\section{(2) OpenEdition}

\section{Journals}

Édition électronique

URL : https://journals.openedition.org/rdr/1653

DOI : $10.4000 /$ rdr. 1653

ISSN : 2534-7462

Éditeur

Presses universitaires de Strasbourg

\section{Édition imprimée}

Date de publication : 2 décembre 2021

Pagination : 129-142

ISBN : 979-10-344-0097-3

ISSN : 2493-8637

\section{Référence électronique}

Thomas Boullu, «L'évolution de la répression judiciaire des abus sexuels commis par des clercs sur les personnes en situation de vulnérabilité (1950-2020)», Revue du droit des religions [En ligne], 12 | 2021, mis en ligne le 02 décembre 2021, consulté le 02 mai 2022. URL : http:// journals.openedition.org/rdr/1653; DOI : https://doi.org/10.4000/rdr.1653

La revue du droit des religions est mise à disposition selon les termes de la Creative Commons Attribution - Pas d'Utilisation Commerciale 4.0 International - CC BY-NC 4.0. 


\section{L'évolution de la répression judiciaire des abus sexuels commis par des clercs sur les personnes en situation de vulnérabilité (1950-2020)}

\section{Thomas BOULLU}

Université de Strasbourg / CNRS, Droit, Religion, Entreprise et Société (DRES)

\section{RÉSUMÉ}

Depuis début 2020, la Commission indépendante sur les abus sexuels dans l'Église catholique a procédé à une vaste enquête au sein des archives ecclésiales et au sein des archives pénales contemporaines. L'étude des données judiciaires recueillies renseigne sur l'évolution de la répression exercée à l'encontre du clergé et permet de mesurer le rapport qu'entretient la société avec les infractions de nature sexuelle. L'analyse de ces archives révèle également certaines spécificités, vraisemblablement propres au monde clérical, qui semblent pouvoir être analysées au regard de l'histoire du catholicisme.

\section{Abstract}

Since early 2020, the Independent Commission on Sexual Abuse in the Catholic Church has been conducting an extensive survey of church archives and contemporary criminal records. The study of the judicial data collected provides information on the evolution of repression against the clergy and allows us to measure the relationship that society has with offences of a sexual nature. The analysis of these archives also reveals certain specificities, probably specific to the clerical world, which seem to be analyzable in the light of the history of Catholicism. 
$\mathrm{L}$ a question des abus sexuels commis par des membres du clergé catholique occupe aujourd'hui une place importante dans le débat public. Depuis les premières révélations aux États-Unis dans les années 1980, le problème de la pédophilie dans l'Église s'est progressivement exporté à l'ensemble du monde catholique. Les scandales se sont multipliés et la couverture médiatique s'est déplacée du suivi d'affaires individuelles judiciarisées au traitement du caractère systémique de la pédophilie dans l'Église ${ }^{1}$. Face à cet emballement médiatique, plusieurs commissions d'enquête ont successivement été mises en place dans les pays anglo-saxons et dans certains États de l'Europe continentale $^{2}$. En France, la Commission indépendante sur les abus sexuels dans l'Église a été créée en novembre 2018 et placée sous la présidence de Jean-Marc Sauvé. Au-delà de la question centrale de la pédophilie, elle a élargi son domaine de recherche à l'ensemble des infractions sexuelles commises depuis 1950 sur des personnes en situation de vulnérabilité ${ }^{3}$. Cette dimension historique permet d'étudier l'histoire des abus sexuels au regard

1. Au-delà des articles de journaux, plusieurs journalistes français ont publié des ouvrages, V. D. Gastaldi, M. Martiniere, M. Périsse, Église, la mécanique du silence, Paris, J.-C. Lattès, 2017; I. DE Gaulmyn, Histoire d'un silence, Paris, Seuil, 2016; M.-C. TABET, Grâce à Dieu, c'est prescrit, Paris, Robert Laffont, 2017. Sur le traitement médiatique des affaires d'abus sexuels dans l'Église, V. O. Bobineau, C. Lalo, J. Merlet, Le sacré incestueux. Les prêtres pédophiles, Paris, Desclée de Brouwer, 2017, p. 7-10.

2. Les premières commissions sont issues des pays anglo-saxons. Pour les États-Unis, V. JohN Jay College of Criminal Justice, The Nature and Scope of the Problem of Sexual Abuse of Minors by Priests and Deacons, Washington, DC, United States Conference of Catholic Bishops, 2004; également K.-J. Terry, The Causes and Context of Sexual Abuse of Minors by Catholic Priests in the United States, 1950-2010, Washington, DC, United States Conference of Catholic Bishops, 2011. Pour l'Irlande, V. le rapport Ryan, Commission to Inquire on Child Abuse Report, vol. III, 2009. Pour l'Australie, Royal Commission into Institutional Responses to Child Sexual Abuse, Analysis of Child Sexual Abuse Made With Respect to Catholic Church Institutions in Australia, 2017. Dernièrement, plusieurs commissions ont également été constituées en Europe continentale. Pour les Pays-Bas, W. DeEtman, Seksueel misbruik van en geweld tegen meisjes in de rooms-katholieke Kerk een vervolgonderzoek, 2013; W. Langelanda, A. W. Hoogendoorn, D. Mager, J. H. Smit, N. Draijer, «Childhood sexual abuse by representatives of the Roman Catholic Church: A prevalence estimate among the Dutch population", Child Abuse \& Neglect, vol. 46, 2015, p. 67-77. Pour l'Allemagne, Sexueller Missbrauch an Minderjährigen durch katholische Priester, Diakone und männliche Ordensangehörige im Bereich der Deutschen Bischofskonferenz, 2018. Pour la Belgique, Chambre des RePrÉsentants De BelgiQue, Le traitement d'abus sexuels et de faits de pédophilie dans une relation d'autorité, en particulier au sein de l'Église, Rapport fait au nom de la Commission spéciale relative au traitement d'abus sexuels et de faits de pédophilie dans une relation d'autorité, en particulier au sein de l'Église, par Mmes S. De Wit et M.-C. Marghem, et MM. R. Terwingen et R. Landuyt, 31 mars 2011.

3. La notion de personne vulnérable est entendue par la commission dans son sens large. Elle intègre les mineurs, les majeurs protégés ainsi que toutes les personnes qui «dans le cadre d'une relation de hiérarchie, d'autorité, d'accompagnement spirituel ou d'emprise, se sont trouvées engagées dans une relation à caractère sexuel non librement consentie»: interview du président J.-M. SAUvé in C. Chambraud, «Abus sexuels dans l'Église française: la commission Sauvé lance son appel à témoignages», Le Monde, 3 juin 2019. 
des mutations qui traversent l'Église catholique et la société française depuis la seconde moitié du $\mathrm{xx}^{\mathrm{e}}$ siècle.

Écrire l'histoire contemporaine de cette forme spécifique de criminalité en France relève de la gageure. L'information, surabondante pour les années récentes ${ }^{4}$, est à l'inverse presque inexistante pour le $\mathrm{xx}^{\mathrm{e}}$ siècle ${ }^{5}$. Afin de pallier cette carence, la constitution d'un matériel scientifique innovant devait donc être envisagée. Les archives judiciaires en forment un des principaux piliers. L'analyse sérielle des procès et des pièces judiciaires apporte une grande diversité d'informations, sur les auteurs, sur les victimes, ainsi que sur les faits qui complètent utilement les données extraites des archives diocésaines ou congrégationistes. Ces documents permettent en outre de mesurer la réponse de l'appareil d'État sur un temps long. La question de la spécificité des abus sexuels dans l'Église, souvent débattue au regard de la figure sacrée du prêtre ou de la réponse des structures ecclésiales, se pose ainsi sous une forme institutionnelle renouvelée. Elle se révèle susceptible d'intégrer des éléments de sécularisation et de laïcisation qui contribuent à l'étude historique du religieux et de l'État.

$\mathrm{Au}$ terme de deux années de campagne d'archives menée par le groupe de recherche socio-historique de la commission Sauvé ${ }^{6}$, un corpus de 494 affaires judiciarisées, dont 299 ayant abouti à une condamnation, a été constitué. L'analyse de ces données judiciaires, qui forme le socle de cette étude, permet d'apporter plusieurs éclaircissements. La répression judiciaire des clercs semble s'inscrire, au moins en partie, dans les grandes évolutions qui traversent le droit des infractions sexuelles au cours de la seconde moitié $\mathrm{du} \mathrm{xx}^{\mathrm{e}}$ siècle. L'étude des condamnations des clercs participe dès lors à mettre en lumière l'émergence d'un mouvement global de répression des infractions

4. Outre la presse, on peut citer M.-J. THIEL, L'Église catholique face aux abus sexuels sur mineurs, Paris, Bayard, 2019; O. Bobineau, op. cit.

5. Deux ouvrages récents procèdent toutefois à une analyse historique, V. M. SÉvEGRAND, Vers une Église sans prêtres. La crise du clergé séculier en France (1945-1978), Rennes, Presses universitaires de Rennes, 2006, p. 73 et p. 93-100 et surtout C. Langlois, On savait, mais quoi? La pédophilie dans l'Église catholique, Paris, Seuil, 2019. Pour le Xix siècle, on peut signaler A. Philibert, Des prêtres et des scandales, Paris, Cerf, 2019; M. Angenot, «Monstres en soutane», Pop-en-Stock, 13 avr. 2019: http://popenstock.ca/monstres-ensoutane [consulté le 2 juin 2021] ; et de manière plus large, G. BERTRIN, « De la criminalité en France dans les congrégations, le clergé et les principales professions libérales», Le Correspondant, 25 janv. 1901.

6. Cette équipe, dirigée par le professeur Philippe Portier, est constituée par l'historien Paul Airiau, par la politiste Anne Lancien et par l'historien du droit Thomas Boullu. Les données sont principalement issues des archives du ministère de la Justice, des parquets, des Archives nationales, des archives départementales et, dans certains cas, des archives de l'Église. Certaines affaires sont encore en cours de transmission auprès des services de la commission et feront l'objet d'une analyse ultérieure. 
sexuelles (1). L'analyse fine des dossiers révèle toutefois certaines spécificités qui attestent une déclinaison particulière de la mise en œuvre de la justice pénale à l'encontre des ecclésiastiques. Ces éléments semblent dépendre, en moins en partie, des transformations que connaît l'Église catholique depuis 1950. L'évolution de la répression judiciaire paraît dès lors pouvoir être étudiée au regard de l'histoire du catholicisme en France (2).

\section{L'INSCRIPTION DE LA RÉPRESSION JUDICIAIRE DES CLERCS DANS L'ÉVOLUTION DE LA RÉPRESSION DES ABUS SEXUELS}

L'étude de la législation pénale du $\mathrm{xx}^{\mathrm{e}}$ siècle révèle l'existence de deux mouvements ${ }^{7}$. Jusque dans les années 1980, le droit en vigueur est presque exclusivement l'héritage des réformes du XIX ${ }^{\mathrm{e}}$ siècle. Les infractions de viol, d'attentat à la pudeur, avec ou sans violence, d'outrage public à la pudeur ou encore d'excitation de mineurs à la débauche structurent l'action publique ${ }^{8}$. À l'exception de l'ordonnance du 2 juillet 1945 rehaussant la majorité sexuelle à l'âge de 15 ans, les réformes sont rares et témoignent un relatif désintérêt des pouvoirs publics dans la lutte contre les infractions sexuelles ${ }^{9}$.

À compter de la fin des années 1980, l'opinion publique se saisit des questions de criminalité sexuelle et l'abus sexuel contre l'enfant devient la transgression majeure. Le pédophile est perçu comme «le nouveau monstre $\mathrm{du} \mathrm{xx}^{\mathrm{e}}$ siècle $^{10}$ » à l'encontre duquel une réponse pénale exemplaire doit s'appliquer. La loi de 1989 sur la protection de l'enfance assouplit les règles

7. Sur l'histoire contemporaine des infractions sexuelles, V. prioritairement A.-C. AMBROISERENDU, Histoire de la pédophilie (XIX'-XXI ${ }^{e}$ siècle), Paris, Fayard, 2014; G. VIGARELlO, Histoire du viol (XVI $-X X^{e}$ siècle), Paris, Seuil, 1998.

8. La mise en place de l'arsenal législatif relatif aux abus sexuels sur mineurs survient successivement en 1810, 1832, et en 1863, V. A.-C. Ambroise-Rendu, «Punir les pédophiles?», in F. Chauvaud (dir.), Le droit de punir du siècle des Lumières à nos jours, Rennes, Presses universitaires de Rennes, 2012, p. 143-156 et J.-P. Alline, «Punir ou éliminer? Deux siècles de pénalisation de l'abus sexuel», id., p. 157-173. L'excitation de mineurs à la débauche suit un développement particulier, principalement jurisprudentiel et dans le flou de la loi pénale, sur ce point, V. H. Duffuler-Vialle, "Les sexualités des mineurs sous le contrôle du juge pénal aux XIX et $\mathrm{Xx}^{\mathrm{e}}$ siècles. L'interprétation jurisprudentielle de l'article 334 du Code pénal - le délit d'excitation de mineurs à la débauche (1810-1942) », Criminocorpus, L'enfance au tribunal. Enjeux historiques, perspectives contemporaines, 2020 : https://journals.openedition.org/criminocorpus/6974 [consulté le 2 juin 2021].

9. L'âge est successivement relevé de 11 ans en 1832 à 13 ans en 1863, puis à 15 ans en 1945, V. ordonnance $\mathrm{n}^{\circ}$ 45-1456: JO, 3 juill. 1945, p. 4010.

10. A. Darsonville, J. Leonhard, «Préface», in A. Darsonville, J. Leonhard (dir.), La loi pénale et le sexe, Nancy, Presses universitaires de Nancy, 2015. L'abus sexuel sur mineur est devenu «le point ultime du mal», selon G. VigareLlo, op. cit., p. 7. 
de prescription et initie un vaste mouvement de réformes ${ }^{11 .}$ Le Code pénal de 1992 remplace l'attentat à la pudeur par les infractions d'agression sexuelle et d'atteinte sexuelle et le délit d'excitation des mineurs à la débauche par celui de corruption de mineurs. Le nouvel article 227-23 du Code pénal permet dorénavant de réprimer la détention, l'exploitation et la diffusion de matériels pédopornographique ${ }^{12}$. L'ensemble de ces infractions fait l'objet de nombreuses modifications conduisant à aggraver progressivement l'échelle des peines auxquelles s'exposent les auteurs ${ }^{13}$. En définitive, un arsenal répressif particulièrement fourni vient sanctionner les infractions sexuelles. Parfois dénoncé comme révélant un phénomène d'inflation législative, il atteste la réception par le législateur d'un changement majeur des mentalités ${ }^{14}$.

L'évolution du nombre de condamnations des clercs pour des abus sexuels sur des personnes en situation de vulnérabilité présente une courbe relativement conforme à celle de l'évolution de l'appareil législatif répressif. Le temps du silence, des années 1950 aux années 1980, précède le temps de la répression, vraisemblablement initié par les réformes de 1989 et de 1992. À l'intérieur de ces deux principaux ensembles, plusieurs variations doivent être relevées. De 1950 à 1990, le nombre de condamnations passe de 54 pour les années 1950 et 1960 à 15 pour les années 1970 et 1980. Ce phénomène de repli s'explique par l'importante diminution des prêtres en exercice en France ${ }^{15}$, mais s'inscrit surtout dans une période de relativisation de la gravité de la pédophilie au sein de la société française. La «plaidoirie pédophile ${ }^{16}{ }$ initiée par certains intellectuels ${ }^{17}$ au nom de la liberté des

11. L. no 89-487, 10 juill. 1989. Sur l'édiction de cette loi, V. A.-C. Ambroise-Rendu, Histoire..., op. cit., p. 201-204. Sur l'arsenal législatif en matière d'infraction sexuelle, V. P. ConTE, Droit pénal spécial, Paris, LexisNexis, 6e éd. 2019, p. 169-202.

12. La loi du $1^{\text {er }}$ février 1994 est votée en urgence juste avant la publication du nouveau Code pénal à la suite de l'affaire Patrick Tissier. V. A.-C. Ambroise-Rendu, «Punir les pédophiles? », art. cit., p. 149.

13. De 1994 à 2020, l'article 227-23 du Code pénal sur la pédopornographie est modifié à sept reprises.

14. J. LeOnhard, «Mineur et sexualité en droit pénal: de la profusion des lois à la confusion du droit », in B. Mallevaey, A. Fretin (dir.), L'enfant et le sexe, Paris, Dalloz, 2021, p. 131-140; Ch. LAZERGES, «Politique criminelle et droit de la pédophilie», RSC 2010, p. 725-741; A. DARSONVILLE, «Le surinvestissement législatif en matière d'infractions sexuelles», Archives de politique criminelle, no 34, 2012/1, p. 31-43.

15. Les ouvrages sont nombreux sur la crise sacerdotale qui traverse l'Église au cours de la seconde moitié du $\mathrm{XX}^{\mathrm{e}}$ siècle, V. principalement M. SÉVEGRAND, op. cit.

16. A.-C. Ambroise-Rendu, Histoire..., op. cit., p. 163-188.

17. On pense notamment aux propos et aux écrits de Gabriel Matzneff actuellement médiatisés; V. également les articles de divers intellectuels parus dans la revue Le petit Gredin: Journal du Groupe de recherche pour une enfance différente. 
mœurs coïncide avec un recul de la répression qui, au-delà de la question du clergé, se confirme dans l'ensemble des statistiques judiciaires ${ }^{18}$.

À l'inverse, de 1990 à aujourd'hui, le nombre de clercs condamnés pour abus sexuels sur personnes en situation de vulnérabilité augmente de manière très sensible. Conforme à l'évolution du code, cette augmentation est également parallèle à celle des condamnés pour abus sexuels sur mineurs dans l'ensemble de la société française ${ }^{19}$. Dans les deux cas, les condamnations atteignent leur pic dans les années 2000 puis connaissent une légère diminution au cours des années 2010. Le nombre de classements sans suite suit un mouvement inverse puisqu'il est multiplié, au cours de la même période, par plus de deux. Cette baisse relative des condamnés jointe à l'augmentation sensible des classements sans suite serait la traduction, au lendemain de l'affaire d'Outreau, d'une plus grande prise en compte par les magistrats des risques d'erreurs judiciaires. À l'issue du verdict d'acquittement général prononcé en 2005 par la cour d'appel de Paris au bénéfice de six accusés, une partie du monde de la robe se positionne contre une tendance au tout répressif. Le Syndicat de la magistrature exhorte le législateur à respecter les principes de l'État de droit et suggère d'« entrer en résistance ${ }^{20} »$. La crainte du retour des «sorcières de Salem ${ }^{21}$ » semble expliquer l'augmentation des acquittements, des classements sans suite ou des relaxes également constatée dans les statistiques judiciaires générales dressées par la Chancellerie ${ }^{22}$. Outre l'étude des condamnations et des classements sans suite, l'évolution de la sévérité des peines prononcées à l'encontre des clercs rejoint ces évolutions générales. Les peines de prison se multiplient et s'allongent entre 1990 et

18. Le nombre total de condamnations pour abus sexuel sur mineurs pour les décennies 1970 et 1980 est en moyenne trois fois moindre que celui des années 1950 et 1960, V. A.-C. Ambroise-Rendu, Histoire..., op. cit., p. 277-278.

19. Le nombre de condamnés annuel moyen est respectivement de 1586 pour les années 19861990, 2543 de 1991 à 1995, 4484 de 1996 à 2000, 4784 de 2001 à 2005 et 4530 de 2006 à 2010. Données extraites de A.-C. Ambroise-Rendu, L'histoire..., op cit., p. 278.

20. «Entrer en résistance», in E. Alt (dir.), Le sexe et ses juges, Paris, Syllepses, 2006, p. 166167. V. également E. Alt, «Outreau: une justice très ordinaire», in E. Alt (dir.), Le sexe et ses juges, op. cit., p. 17-21. Plus récemment, J. LEONHARD, «Représentations du Droit sur la sexualité du mineur: droit et non droit», in J. Leonhard, V. Olech (dir.), Violences sexuelles: entre vérités et mensonges, Nancy, Presses universitaires de Nancy, 2019.

21. A. Garapon, D. Salas, Les nouvelles sorcières de Salem: leçons d'Outreau, Paris, Seuil, 2006. L'expression renvoie aux procès pour sorcellerie conduits dans la région de Salem à la fin du XVII siècle et ayant abouti à l'exécution de vingt personnes.

22. Selon Youssef Badr, porte-parole de la Chancellerie, l'affaire Outreau a conduit à «renforcer les exigences probatoires dans les enquêtes dans lesquelles les paroles de la victime et de l'auteur s'opposent »: J.-B. JACQUIN, «En dix ans, le nombre de personnes condamnées pour viol a chuté de $40 \%$ », Le Monde, 14 sept. 2018. 
$2010^{23}$ puis connaissent un infléchissement qui laisse supposer un assouplissement de la mise en ouvre de la loi pénale ${ }^{24}$.

Évolution de la répression judiciaire des clercs accusés d'abus sexuels sur personnes vulnérables (1950-2020)

(Graphique réalisé sur la base de 382 données)

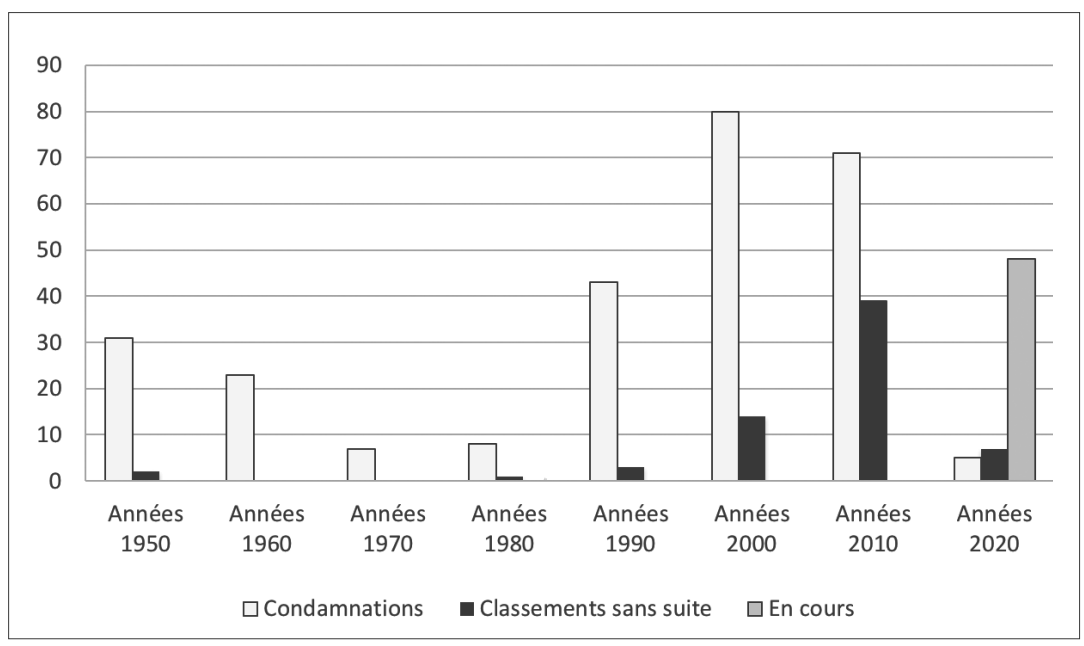

En définitive, l'étude des condamnations des clercs pour abus sexuel sur personne vulnérable offre un regard particulier sur l'évolution générale du droit pénal de la sexualité. Les changements des mentalités influencent fortement l'œuvre législative; l'ensemble conduisant à dessiner les principales tendances de la répression judiciaire. Dans ce contexte, le statut des prêtres et des religieux condamnés ne semble pas conduire à une quelconque spécificité.

\section{L'INSCRIPTION DE LA RÉPRESSION JUDICIAIRE DES CLERCS DANS L'ÉVOLUTION DU CATHOLICISME EN FRANCE}

La consultation des archives judiciaires atteste la présence de certaines particularités vraisemblablement propres au monde clérical. La répartition géographique des condamnations par ressorts de cours d'appel se révèle atypique. Elle rappelle, dans ses grandes lignes, la Carte religieuse de la

23. Pour les années 1950, la peine moyenne par condamné est de 4,2 mois. Dans les années 2000, elle passe à 6 mois.

24. Le nombre de peines fermes identifiées dans nos sources passe de 35 dans les années 2000 à 24 dans les années 2010. 
France rurale dressée par le chanoine Boulard en $1947^{25}$ ou encore, de manière assez similaire, la Carte de la pratique pascale de $1966^{26}$. L'ensemble de la Bretagne, le nord de la France, les anciennes terres concordataires, la région de Besançon ainsi que celle de Lyon composent un arc de cercle de catholicité bien identifié où le nombre de condamnations est important. Les zones frontalières semblent, de manière générale, davantage concernées par la thématique des abus sexuels. Cette constatation entre en résonnance avec les écrits du doyen Gabriel Le Bras qui rappelait que «le christianisme résiste aux frontières ${ }^{27} »$. La diagonale du vide dont rend compte la carte Boulard figure également dans la carte des condamnés pour abus sexuels. De la Meuse à l'Ariège, les condamnations sont plus faibles, voire inexistantes, comme dans le ressort de la cour d'appel de Limoges. Plusieurs ensembles géographiques se démarquent toutefois de la carte Boulard. L'ouest de la Normandie, le Pays basque ou encore la Savoie semblent sous-représentés. À l'inverse, la région parisienne ainsi que le bassin méditerranéen concentrent un nombre important de condamnations alors qu'ils ne sont traditionnellement pas présentés comme des terres de pratique catholique. La surreprésentation de la capitale pourrait s'expliquer par l'importance de son presbyterium qui devient, au cours de la seconde moitié du $\mathrm{xx}^{\mathrm{e}}$ siècle, de plus en plus important. Dans le sud-est de la France, les condamnations que nous avons pu relever sont relativement récentes. La surreprésentation de cet espace géographique pourrait dès lors servir le propos d'Hervé Le Bras et d'Emmanuel Todd convenant d'une disparition progressive de la réalité Boulard au fil des années, parallèlement au recul du catholicisme ${ }^{28}$. Cette approche est confirmée par le nombre plus important de condamnations rendues au sein des territoires catholiques ${ }^{29}$ dans les années 1950 à 1970 par rapport aux périodes les plus contemporaines. En définitive, l'évolution de l'histoire du catholicisme renseigne sur l'évolution de la répression

25. F. Boulard, «Carte religieuse de la France rurale (novembre 1947)», Cahiers du clergé rural, nov. 1947, p. 403. La carte Boulard se retrouve dans d'autres études, V. principalement T. TACKeTt, La Révolution, l'Église, la France, Paris, Cerf, 1986 et plus récemment, G. Cuchet, «La carte du chanoine Boulard», L'Histoire, n 443, janv. 2018, p. 73: https:// www.lhistoire.fr/la-carte-du-chanoine-boulard-0 [consulté le 2 juin 2021].

26. F. Boulard, Premiers itinéraires en sociologie religieuse, Paris, Éditions ouvrières, 1966, p. 13.

27. G. LE BRAS, «Commentaires sociologiques des cartes religieuses de la France», Lumen vitae, $\mathrm{n}^{\circ} 3$ 3, 1948, p. 637, cité in G. CUCHET, Comment notre monde a cessé d'être chrétien. Anatomie d'un effondrement, Paris, Seuil, 2018, p. 55.

28. H. Le Bras, E. Todd, Le mystère français, Paris, Seuil, 2013 p. 79; G. CuChet, Comment notre monde..., op. cit., p. 82-85.

29. Sur 43 condamnations pour lesquelles les tribunaux sont connus entre 1950 et 1960 , 29 sont rendues dans des territoires que le chanoine Boulard identifie comme de terres catholiques, soit près de $75 \%$. 
judiciaire dont la spécificité tend progressivement à s'estomper au profit d'une uniformisation.

Carte de la pratique religieuse dans la France rurale, établie par F. Boulard en 1947

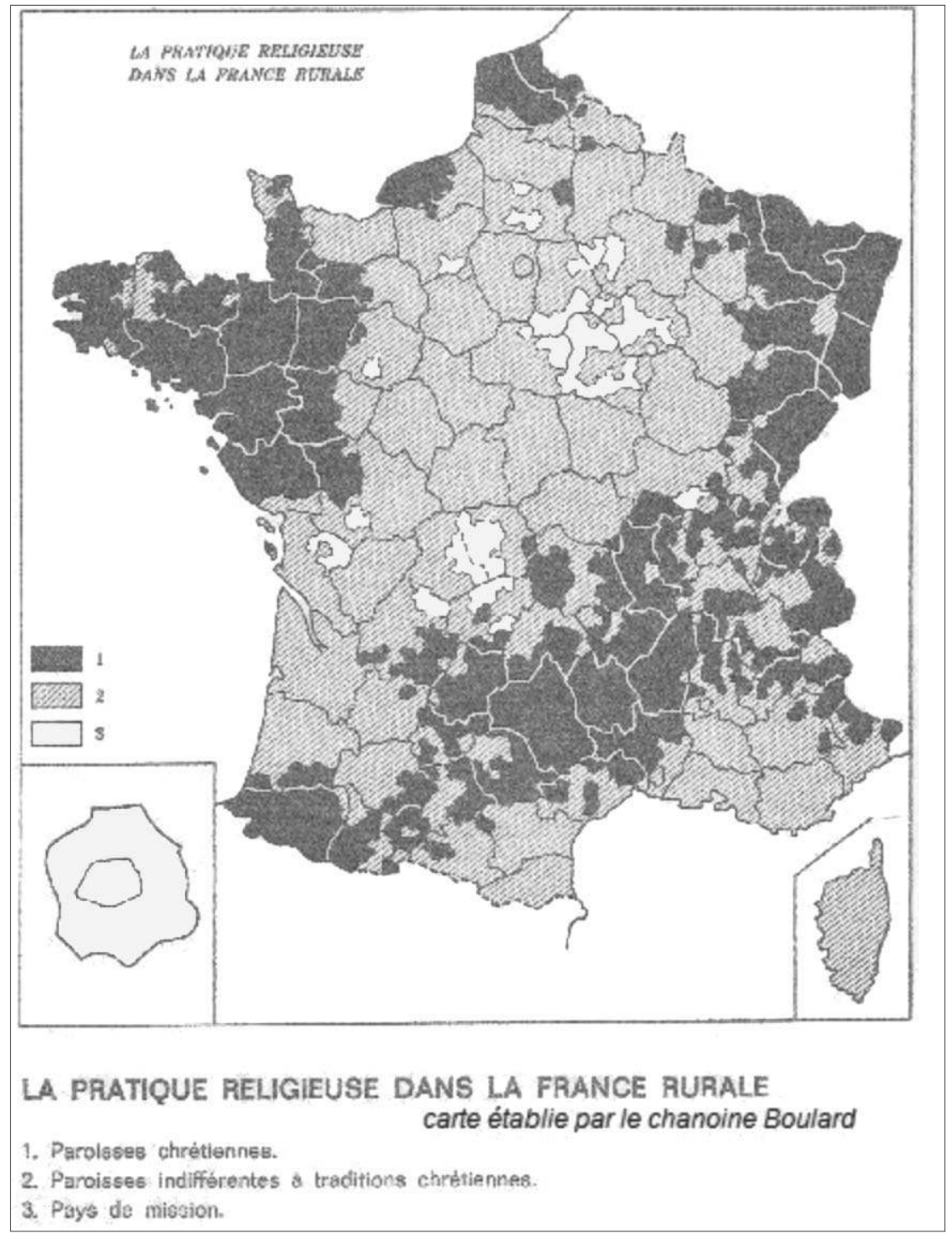

(Malgré tous nos efforts, les organismes ou ayants droit de certains documents iconographiques reproduits dans le présent ouvrage n'ont pu être contactés ou n'ont pas répondu à nos sollicitations. Nous tenons à leur disposition les droits usuels en notre comptabilité.) 


\section{Répartition géographique des condamnations de clercs pour abus sexuels sur des personnes en situation de vulnérabilité par ressorts de cours d'appel (1950-2020) ${ }^{30}$}

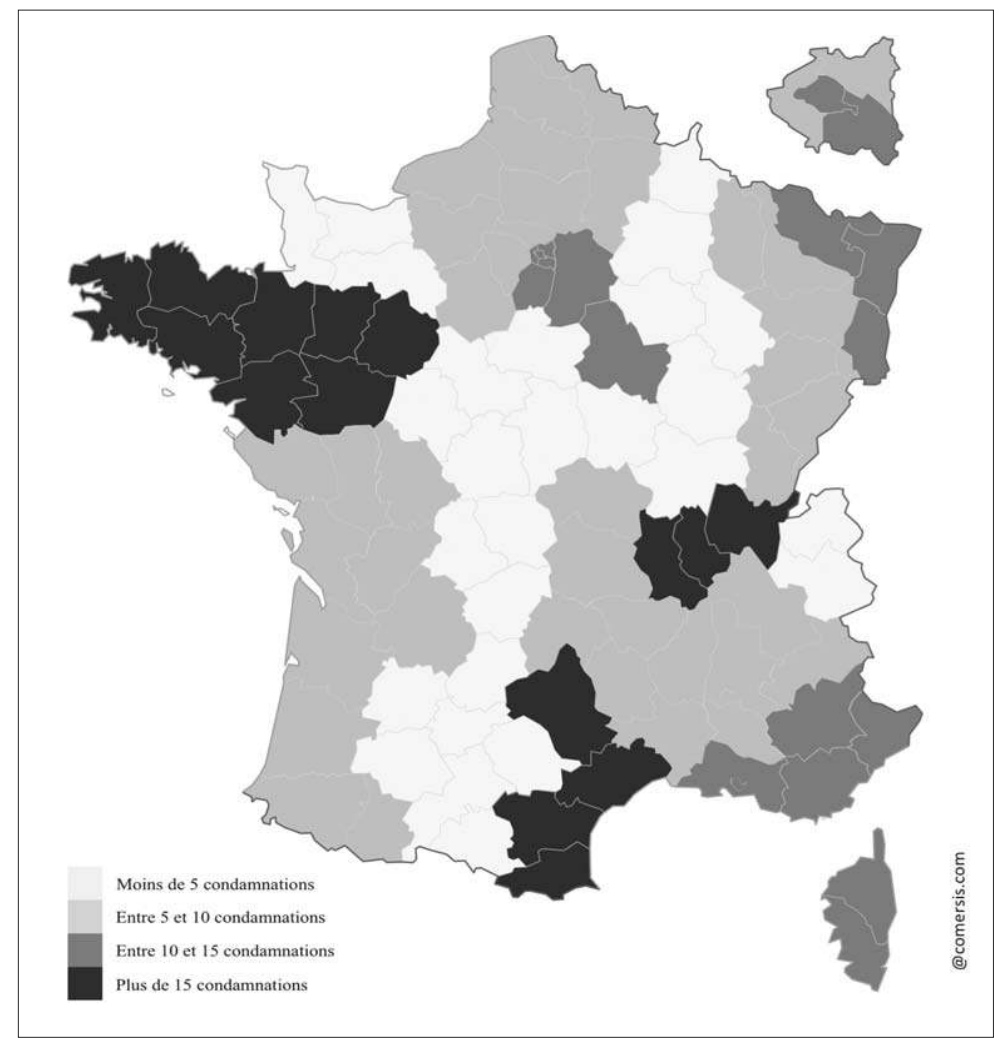

L'étude de l'évolution de l'âge moyen des condamnés semble également pouvoir être étudiée au regard de l'histoire du catholicisme. L'âge moyen des clercs condamnés se révèle sensiblement plus élevé que celui identifié au sein de la population française. Alors que les infractions sexuelles sur mineurs concernent classiquement des trentenaires ${ }^{31}$, l'âge moyen des ecclésiastiques

30. Répartition géographique établie sur un total de 214 condamnations. Les ressorts des cours d'appel de Metz et de Colmar ainsi que les ressorts des cours d'Aix-en-Provence et de Bastia ont été regroupés, conformément aux recommandations de la commission, afin de ne pas permettre d'identifier le nombre d'auteurs au sein d'un unique diocèse.

31. L'enquête Ipsos réalisée en 2019 auprès de 502 victimes estime que les auteurs ont «en moyenne 30 ans »: Violences sexuelles dans l'enfance, enquête après des victimes, sept. 2019, p. 27 : https://www.ipsos.com/fr-fr/10-ans-lage-moyen-des-premieres-violences-sexuelles-0 [consulté le 2 juin 2021]. Le travail de Véronique Le Goaziou sur 488 auteurs jugés pour viols entre 1998 et 2008 révèle une moyenne d'âge de 31 ans au moment du passage à l'acte. Au sein de la catégorie des viols familiaux, presque exclusivement commis à 
s'élève, dans nos archives, à plus de 55 ans $^{32}$. Cette tendance se renforce progressivement, l'âge des condamnés reculant au fil des décennies. Cette évolution s'explique, au moins en partie, par le vieillissement général du corps clérical. Les candidats au sacerdoce se présentent de plus en plus tardivement au grand séminaire et, surtout, partent de plus en plus tard en raison de l'absence de remplaçant. En France, le prêtre appartient très souvent «au quatrième âge», convient Céline Béraud qui précise qu'un prêtre sur deux a plus de 75 ans dans les années $2010^{33}$. L'augmentation de l'âge des clercs condamnés révélerait donc la mutation sociale du clergé depuis la seconde moitié du $\mathrm{Xx}^{\mathrm{e}}$ siècle.

Les archives révèlent également l'évolution des rapports qu'entretiennent certains évêques ou certains supérieurs de congrégations avec les services de la justice. Dans plusieurs cas, des négociations et des ententes entre parquetiers et responsables ecclésiastiques permettent au clerc d'éviter une judiciarisation du litige. De telles situations, sans être excessivement nombreuses, représentent cependant une quantité non négligeable dans les premières années visées par la présente étude. Dans les années 1950, neuf affaires, soit un tiers de l'ensemble des condamnations identifiées pour cette décennie, sont étouffées avec la complicité de représentants des pouvoirs publics. La principale motivation semble être la sauvegarde de la réputation de l'Église catholique. L'ambition première est «d'éviter le scandale», déclare un président de tribunal en 1950 informé de la commission d'attentats à la pudeur par un religieux. Dans une autre situation, il est essentiel «de ne pas rendre l'affaire publique» convient le procureur qui se présente comme « désireux d'arranger les choses». En 1951, un évêque reconnaît que le «Haut commissaire, le Secrétaire général, le Procureur général [...] tout le monde a gardé la plus grande discrétion » ce qui a permis d'éviter l'esclandre. Dans une affaire d'attouchements commis dans le Languedoc à la fin des années 1950, «une enquête a eu lieu» informe le procureur. "Je me suis efforcé d'en circonscrire la portée», précise-t-il à son évêque «et je tâcherai d'éviter un scandale dont nous avons si peu besoin ». Dans plusieurs situations, les magistrats sollicitent la mutation des prêtres et des religieux accusés d'avoir commis des abus. Ils participent ainsi aux politiques de déplacements des prêtres

l'encontre des mineurs, la moyenne est de 35 ans: V. Le GoAziou, Le viol, aspects sociologiques d'un crime, Paris, La Documentation française, 2011, p. 41.

32. La statistique nationale est en partie induite par le fait que la majorité des viols sur mineurs sont également commis par des mineurs.

33. C. BÉRAUD, «Le catholicisme français à l'épreuve du vieillissement », in Y. TRANVOUEZ (dir.), La décomposition des chrétientés occidentales (1950-2010), Brest, Ouestélio, 2013, p. 58. 
dont le phénomène est désormais connu ${ }^{34}$. Toujours dans les années 1950 , un procureur ordonne «que l'autorité ecclésiastique déplace immédiatement l'intéressé » sous peine d'engager des poursuites. Dans une autre affaire, le parquet conditionne l'interruption des poursuites à un «changement d'affectation dans un poste où il ne sera plus en contact avec des enfants». Les termes employés par les magistrats se ressemblent d'une correspondance à une autre et attestent d'une philosophie commune. En 1958, un parquetier «suggère d'envisager immédiatement le changement du vicaire de [...]. C'est urgent, et cette mesure me permettra peut-être d'éviter des poursuites ». "Suis mon conseil et attendons l'oubli», recommande vivement le procureur à l'évêque. Au-delà de ces affaires qui n'arrivent pas jusqu'au prétoire, plusieurs archives rendent compte de magistrats qui communiquent des informations aux évêques sur des dossiers en cours. Ils manquent ainsi à leur devoir de réserve et, pour certains, violent le secret de l'instruction et de l'enquête. Ces infractions, moins graves que les précédentes, continuent d'être rencontrées tardivement dans les archives, parfois encore au $\mathrm{XxI}^{\mathrm{e}}$ siècle $^{35}$.

Même s'il ne faut pas exagérer la proportion de ces affaires, l'étude confirme l'existence de liens privilégiés entre une partie du monde de la robe et celui de l'aube. Ces liens s'expliquent par la foi catholique que partagent ces parquetiers. Un procureur reconnaît que «c'est son devoir de chrétien » d'informer l'évêque des infractions commises par un prêtre. En retour, l'évêque lui adresse «sa paternelle bénédiction» et lui exprime son plaisir de l'avoir vu lors de sa "cérémonie d'intronisation». Dans une autre affaire datant de 1959, l'adhésion du substitut général à la religion catholique explique, selon l'évêque, que ce dernier soit intervenu directement pour «faire le silence sur cette affaire». "C'est un bon chrétien!» affirme un évêque du sud de la France pour illustrer la qualité des relations qu'il entretient avec le parquet général. De telles archives rappellent les études entreprises par Jean-Pierre Royer sur la magistrature catholique sous la

34. La pratique est identifiée dans les rapports des commissions étrangères. Pour la France, on peut citer l'intervention de Mgr Ravel, «Une affaire des années 1950 » présentée dans le cadre du colloque Agressions sexuelles sur mineurs par des cadres religieux, qui s'est tenu les 3-4 déc. 2019 à l'Université de Strasbourg.

35. Cela semble être le cas d'un procureur général qui, au xxI siècle, tient fidèlement informé son évêque: «En vous priant de trouver copie du rapport que vient de m'adresser le Procureur de la République de [...] que j'avais aussitôt interrogé. Les services de police se sont abstenus de faire apparaître dans leurs messages et synthèses, la qualité du père $[\ldots]$. Dès lors que la victime demeurera discrète, on peut espérer une absence de médiatisation. Je continuerai à suivre personnellement cette affaire et demeure à votre disposition. Avec l'expression de mes sentiments fidèlement dévoués.» 
troisième République ${ }^{36}$. Les documents indiquent en effet que le lien historique entre l'Église et la justice conserve certains vestiges au sein d'une société judiciaire laïcisée, mais de tradition catholique ${ }^{37}$.

Progressivement, au cours du $\mathrm{xx}^{\mathrm{e}}$ siècle, ce type d'archives se font de plus en plus rares. L'influence de l'Église catholique tend à se réduire et le monde de la magistrature est traversé, à l'instar de la société, par le processus de sécularisation. Dans une note privée datée de 2001, un magistrat haut placé déconseille dorénavant aux évêques de contacter le parquet lorsqu'un prêtre est mis en cause sauf «relations privilégiées et très personnelles ${ }^{38}$ ». Ce moment de rupture précède l'instauration d'une soumission plus généralisée des autorités ecclésiastiques à la justice civile. Une nouvelle forme de collaboration s'instaure, au service de la transparence, qui remplace la culture du secret. Certains parquets, à l'instar de celui de Paris, établissent des conventions avec les diocèses qui s'engagent à délivrer toute information paraissant vraisemblable en matière d'infraction sexuelle. La mise en place d'un cadre institutionnel original atteste ainsi une nouvelle spécificité propre à l'Église susceptible d'exercer une incidence sur la répression judiciaire.

Tous les éléments développés dans cette étude méritent un travail d'analyse complémentaire auquel viendront s'ajouter les archives qui continuent à l'heure actuelle d'être transmises à la Commission indépendante sur les abus dans l'Église. Au-delà des tendances que révèlent ces premiers résultats, une exploration plus fine des dossiers de procédure et plus particulièrement des affaires d'assises pourra être entreprise. Certains éléments pourraient être étudiés plus précisément à l'instar du taux de récidive légal, établi à 7,2 \% pour les clercs selon nos archives, soit nettement en dessous des estimations

36. Au lendemain des grandes épurations de la magistrature, le «juge fonctionnaire », davantage sensible aux idées républicaines et laiques, succède au «juge notable» reflet d'une société tournée vers le monde rural et la religion catholique: J.-P. ROYER, Juges et notables au XIX siècle, Paris, PUF, 1982, p. 377-387; J.-P. Royer, J.-P. Jean, B. Durand, N. Derasse, B. Dubois, Histoire de la justice en France, Paris, PUF, 4e éd. 2010, p. 739-749; p. 377387. V. également J.C. FARCY, Les carrières des magistrats (XIX ${ }^{e}-X X^{e}$ siècles). Annuaire rétrospectif de la magistrature, Dijon, Centre Georges Chevrier, Mission de recherche Droit et justice, 2009, p. 5-6.

37. J.-P. ROYER, Juges et notables..., op. cit., p. 384.

38. Archives conservées au Centre national des archives de l'Église de France (CNAEF). Note du 21 juin 2001: "À mon avis et sauf relations privilégiées et très personnelles, qui pourraient rester sous le sceau de la confidence, ne pas chercher, pour l'évêque à prendre contact avec l'autorité judiciaire, que ce soit avec le Procureur de la République ou - et surtout - le Juge d'instruction. Si celui-ci désire entendre l'évêque, il le lui fera savoir. Si, toutefois, une nécessité de contact apparaît comme nécessaire ou indispensable, passer toujours par l'entremise de l'avocat. » 
nationales $^{39}$. Le nombre important de dossiers en cours d'instruction ou d'enquête laisse également penser que les années 2020 pourraient connaître un nouveau regain de condamnations ${ }^{40}$. Cette évolution, si elle se confirme, pourrait en partie être attribuée à la mise en place de la commission Sauvé qui participe, au-delà de l'étude des abus sexuels dans l'Église, à la libération de la parole.

39. En 1982, le taux de récidive en matière d'attentats à la pudeur est de 31 \% selon C. BALIER, A. Ciavaldini, M. Girard-Khayat, Rapport de recherche sur les agresseurs sexuels, Paris, La Documentation française, 1996, p. 213; V. F. DE BruYn, A. Kensey, 50 ans d'études quantitatives sur les récidives enregistrées, Paris, Ministère de la Justice, 2017, p. 36. En 1997, la Direction centralisée de la police judiciaire publie un taux de récidive de $8 \%$ en matière de viols mais de 20,7\% en matière d'attentats à la pudeur, cité in P. BESSOLES, «Le complexe criminogène. Contribution à une psychogenèse de la criminalité sexuelle», Bulletin de psychologie, $\mathrm{n}^{\circ} 481,2006 / 1$, p. 75-80. Les statistiques relatives aux prisonniers libérés de 2002 conviennent d'un taux de récidive de $19 \%$ pour les viols sur mineurs et de $21 \%$ pour les attentats et outrages à la pudeur sur mineurs. Commenté par A. Kensey, "Aménagements de peines et moindre récidive», Criminocorpus, L'aménagement des peines privatives de liberté: l'exécution de la peine autrement, 2013: https://journals.openedition.org/criminocorpus/2489 [consulté le 2 juin 2021]. En 2018, près de $20 \%$ des auteurs d'infraction sexuelle avaient déjà été condamnés au cours des dix dernières années, V. M. Juillard, O. Timbart, «Les condamnations pour violences sexuelles», Infostatjustice, $\mathrm{n}^{\circ} 164,2018, \mathrm{p} .1$.

40. Les dossiers actuellement en cours pour lesquels nous disposons d'informations sont au nombre de 49, soit la moitié des condamnations prononcées pour la décennie 2000-2010. 